\title{
Assessing Perceptions of Broad Consent Concerning Biological Specimen Collection in a Cohort of Young Sexual Minority Men
}

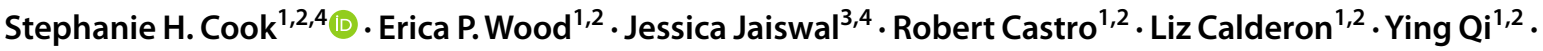 \\ Amanda Llaneza ${ }^{1,2}$
}

Received: 23 April 2019 / Revised: 16 February 2021 / Accepted: 18 February 2021 / Published online: 6 October 2021

(c) The Author(s), under exclusive licence to Springer Science+Business Media, LLC, part of Springer Nature 2021

\begin{abstract}
The use of broad consent to store human biospecimens to be used in future research studies has increased over the years. However, it is currently unknown how young sexual minority men (YSMM) perceive broad consent in these specific types of studies. Therefore, in this study we aimed to determine the extent to which YSMM are comfortable with providing broad consent concerning their identifiable biological specimens to a variety of entities, including external researchers and pharmaceutical companies and to examine the relationship between mistrust based on racial/ethnic identity or sexual orientation and attitudes toward broad consent. YSMM $(N=239)$ ages 24-27 years were recruited from a prospective cohort study in New York City in 2018 to complete a survey assessing attitudes about the use of broad consent concerning biospecimens for secondary research. We found that YSMM were most willing to provide broad consent to the researcher from the study they were enrolled in (85.3\%), other researchers within the same university (82.4\%), and researchers at other universities (74.5\%). Participants were least willing to provide broad consent to government organizations (64.4\%) and pharmaceutical companies (53.8\%). Further, we found that medical mistrust based on racial/ethnic identity or sexual orientation was associated with attitudes toward the use of broad consent. Research institutions should consider modifying consent procedures around the use of broad consent in order to maximize recruitment and retention, especially among minority populations.
\end{abstract}

Keywords Young sexual minority men $\cdot$ Biologics $\cdot$ Ethics $\cdot$ Mistrust $\cdot$ Sexual orientation

\section{Introduction}

Research involving the use of stored human biospecimens (e.g., saliva, blood) from biobanks has grown considerably over the past 30 years (De Souza \& Greenspan, 2013).

Supplementary Information The online version contains supplementary material available at https://doi.org/10.1007/ s10508-021-01964-3.

Stephanie H. Cook

sc5810@nyu.edu

1 Department of Biostatistics, School of Global Public Health, New York University, 715 Broadway, Room 1014, New York, NY 10012, USA

2 Department of Social Behavioral Sciences, School of Global Public Health, New York University, New York, NY, USA

3 Rory Meyers School of Nursing, New York University, New York, NY, USA

4 Center for Health, Identity, Behavior and Prevention Studies, Rutgers University, New Brunswick, NJ, USA
Although originally created to be used only within the scope of a specific research focus, biobanks are increasingly being used in studies that differ in terms of the original research question and/or investigators due to factors such as biotechnological advances and more readily available and reliable collection techniques (Fisher \& Layman, 2018). In turn, research using stored human biospecimens has elicited unique ethical considerations in relation to informed consent from human donors. In 2017, the Office of Human Research Protections (OHRP) updated federal regulations for the protection of human subjects who participate in studies that may collect and store biospecimens (Department of Homeland Security, 2017). The rule went into effect in January 2019. This rule, known as the Common Rule, states that researchers may collect general informed consent for the storage and future use of human biospecimens in secondary research regardless of whether the potential study differs from the focus of the original study or if being conducted by different researchers, if broad consent was properly documented and storage 
provisions to protect confidentiality are adequate (Grady et al., 2015; A Notice of Proposed Rule Making [NPRM], 2016).

Policy surrounding the use of broad consent for secondary research with stored biospecimens continues to be nebulous, and consequently, is applied inconsistently across institutions (Fisher \& Layman, 2018; Rothstein et al., 2016). For instance, the revised Common Rule relies on the assessment of individual Institutional Review Boards (IRB) to define what information should be included on the consent form with respect to storage and future research with stored biospecimens if it meets the informed consent criteria set forth by the OHRP (Fisher \& Layman, 2018). In addition, the updated Common Rule includes a provision for convening a committee for periodic review of the definition of broad consent. Thus, researchers will need to continuously be aware of and adapt to changing definitions of identifiable biospecimens in human subjects research in order to properly utilize broad consent (Fisher \& Layman, 2018). Lastly, the Common Rule stipulates that secondary research with stored biospecimens obtained under the use of broad consent may require only limited IRB review. Nevertheless, the updated Common Rule allows future researchers to be able to use participants' identifiable biospecimens for unspecified research purposes that may not relate to the original study (Menikoff et al., 2017).

An important consideration for researchers and IRBs to take into account is that attitudes that participants have regarding the use of their stored biospecimens in secondary research studies are likely to be impacted by the nature of the study, the type of researchers utilizing their samples, and the characteristics of the participants themselves (De Vries et al., 2016; Grady et al., 2015; Hiratsuka et al., 2012; Sanderson et al., 2017). Moreover, a recent systematic review of broad consent found that many potential participants did not feel that the broad consent procedure was ethically adequate (De Vries et al., 2016). Other studies have further found that perceptions and motivations to participate in research involving the collection of biospecimens differ across populations (Kerath et al., 2013; Michie et al., 2011; Sanderson et al., 2017). Thus, the adoption of broad consent may not be appropriate for every research setting involving the collection and storage of human biospecimens. In particular, racial and ethnic minority populations may hold reservations regarding the use of their biological samples in research due to mistrust of the medical community (Eaton et al., 2015; Hiratsuka et al., 2012). Indeed, there is a longstanding history of racism embedded within medical institutions and research in the U.S. (e.g., the U.S. Public Health Service Syphilis Study at Tuskegee) that has, in turn, negatively influenced willingness to participate in research among minority populations (Katz et al., 2006).
Moreover, the increasing collection and storage of biospecimens from sexual minority men (SMM) for HIVrelated research (e.g., research involving pre-exposure prophylaxis [PrEP]) has increased considerably in recent years (De Souza \& Greenspan, 2013). Research also shows that SMM, especially SMM of color, may also harbor feelings of mistrust in the medical community, which may, in turn, influence willingness to participate in biomedical research (Eaton et al., 2015; Hoyt et al., 2012). Nevertheless, there is a paucity of research that seeks to understand how SMM in general and SMM of color in particular feel regarding the use of broad consent for secondary research using their stored biological data.

Although several studies have found that most individuals would give broad consent for the storage and future use of their biological samples (De Vries et al., 2016; Richter et al., 2018; Sanderson et al., 2017), the current literature consists of sample populations that are predominately white and/or heterosexual and of a wide age range. Thus, it is important that research addresses the ethical concerns in relation to the use of broad consent among diverse populations, including young sexual minority men (YSMM). The current study had two overarching aims in order to address these limitations. The first aim was to examine attitudes about the use of broad consent concerning biospecimens for secondary research among YSMM. More specifically, we sought to explore whether there were differences in attitudes toward broad consent for samples used by different institutions (e.g., university researchers versus pharmaceutical researchers) and/or types of secondary research (e.g., HIV-related research versus cancer research). Second, we sought to understand how mistrust in the medical community based on one's racial/ethnic identity or sexual orientation influences one's attitudes toward providing broad consent. The goal of the study was to gain empirical insight into the views that YSMM hold about the use of broad consent for the use of their stored biospecimens in secondary research.

\section{Method}

\section{Community Advisory Board}

The Principal Investigator (PI; Cook) of the study consulted The Center for Drug Use and HIV Research community advisory board (CAB) in the development of the study and in the interpretation of the study results. The $\mathrm{CAB}$ was comprised of 12 members of the research, service, and other community-based organizations that serve LGBT communities. The PI met with the CAB to receive input on the overarching research questions and study design to 
ensure that the study design was culturally relevant. After completion of data collection, the PI met again with the $\mathrm{CAB}$ to discuss study findings and implications.

\section{Participants}

Participants were recruited from the P18 Cohort Study, an ongoing prospective cohort study that seeks to examine the biological, psychological, and social underpinnings of health and health behaviors among a cohort of YSMM in the greater New York City area as they emerge into adulthood. P18 participants provided biological samples for HIV testing, but samples were immediately destroyed after testing. P18 Cohort participants were recruited for their baseline visits between 2009 and 2011 when they were between the ages of 18-19 years old and have been followed up semi-annually ever since. As of 2018, participants were between the ages of 24-27 years. Full study details of the P18 Cohort Study have been described elsewhere (Halkitis et al., 2013).

Participants for the current study were recruited at one of their regularly scheduled visits in the P18 Cohort Study. A member of the research team read the script introducing the study and, if the participant indicated interest, took them through the informed consent process. The participant then provided written informed consent and took an online survey via Qualtrics® that took an average of $15 \mathrm{~min}$ to complete. All participants were provided with $\$ 5$ as compensation for the completion of the survey. Of the $n=385$ young men in the P18 Cohort who were invited to participate in the current study, $63 \%$ (241) agreed to participate. The study was approved by the New York University IRB. Because two young men were missing data pertaining to race/ethnicity, the final study sample consisted of $N=239$ YSMM. The mean age of the sample was 25.8 years ( $\mathrm{SD}=0.83$, range $24-27$ ). The vast majority $(80.8 \%)$ of the sample identified as gay, while $10.5 \%$ identified as bisexual, $6.7 \%$ identified as "other," and the remaining $2.1 \%$ identified as heterosexual. In terms of education, most participants had at least some college (66\%) while the remaining reported having a high school diploma or GED (34\%). See Table 1 for sociodemographics of the study sample.

\section{Measures}

\section{Sociodemographics}

Participants self-reported all sociodemographic characteristics. Age was calculated by subtracting self-reported date of birth from the date of their survey. Race/ethnicity was ascertained by asking participants if they identified as White, Black, Asian/Pacific Islander, American Indian/Alaska Native, Other, and/or Hispanic/Latino. We collapsed the race/ethnicity variable into the following categories: White,
Table 1 Descriptive statistics $(N=237)$

\begin{tabular}{ll}
\hline & $M(\mathrm{SD}) / \%(N)$ \\
\hline Age & $25.86(.83)$ \\
Race/Ethnicity & \\
White, non-Hispanic & $26.58(63)$ \\
Non-White, non-Hispanic & $44.73(106)$ \\
Other, Hispanic & $28.69(68)$ \\
Education & \\
High school diploma/GED & $33.33(79)$ \\
Some college or more & $66.67(158)$ \\
Sexual orientation & \\
Gay & $80.59(191)$ \\
Bisexual & $10.55(25)$ \\
Other & $8.86(21)$ \\
Broad Consent Institution Types & \\
Current researchers & $3.39(.86)$ \\
Other researchers within this university & $3.31(.91)$ \\
Researchers at other universities & $3.10(1.02)$ \\
Government organizations & $2.82(1.13)$ \\
Pharmaceutical companies & $2.61(1.14)$ \\
Broad Consent Research Scenarios & \\
High blood pressure research & \\
Cancer research & $3.03(.95)$ \\
Vaccination for a common disease & $3.09(.91)$ \\
Vaccination for a less common disease & $3.01(.96)$ \\
Racial/ethnic differences in HIV progression & $3.08(.93)$ \\
Gexual behavior differences in HIV progression & $3.08(.91)$ \\
GBMM & $3.04(.94)$ \\
GBMM - Race/ethnicity & $2.97(.98)$ \\
\hline & $2.24(.78)$ \\
\hline & $2.39(.80)$ \\
\hline
\end{tabular}

${ }^{\mathrm{a}}$ Variables range from 1-4, with higher scores denoting greater trust

${ }^{\mathrm{b}}$ Group-based Medical Mistrust Scale

non-Hispanic; non-White, non-Hispanic, and Hispanic due to a small number of respondents identifying as Black, Hispanic $(n=6)$, Multi-Racial, Hispanic $(n=5)$, and American Indian/Alaska Native $(n=6)$. Respondents self-reported sexual orientation by asking participants if they considered themselves to be $1=$ Heterosexual or straight, $2=$ Gay or lesbian, $3=$ Bisexual, or $4=$ Other. Highest level of education attained was collapsed into $1=$ High school or less versus $2=$ Some college or more categories.

\section{Attitudes Toward Broad Consent}

We developed a set of questions related to attitudes toward the use of broad consent in collaboration with the $\mathrm{CAB}$ involving research with stored biospecimens (see supplement for questionnaire). The questions were preceded with a brief description of broad consent, along with a list of institutions and scenarios in which their biospecimens may be used in secondary research. 
They were asked to indicate how willing they were to provide broad consent for several research scenarios among several institution types (1) the researcher from the current study they were enrolled in, (2) other researchers within this university, (3) researchers at other universities, (4) pharmaceutical companies, and (5) government organizations. The scenarios for secondary research included (1) high blood pressure research; (2) cancer research; (3) vaccination development for common diseases (e.g., the flu); (4) vaccination development for less common diseases (e.g., HIV); (5) for studying racial/ethnic differences; (6) for understanding sexual behavioral differences in disease progression; and (7) for studying genetic differences among populations. Answers fell on a 4-point Likert-scale, ranging from 1 "Not At All Willing" to 4 "Very Willing." We dichotomized this variable to $0=$ "Not At All Willing" vs. $1=$ "Willing" due to the small amount of variation among the "Willing" groups. The scale showed excellent internal consistency among each of the seven scenarios ( $\alpha>0.96$ for each scenario). Table 1 displays average willingness to provide broad consent for each institution type and each scenario.

\section{Medical Mistrust: Race/Ethnicity}

The Group-Based Medical Mistrust (GBMM) scale (Thompson et al., 2004) was used to assess mistrust in the medical community due to one's race and/or ethnicity. The scale consists of 12 items that fall on a 5-point Likert scale from 1 "Strongly Disagree" to 5 "Strongly Agree." Example items include "People of my ethnic group cannot trust doctors and health care workers" and "I have personally been treated poorly or unfairly by doctors or health care workers based on my ethnicity." The scale showed high internal consistency with our study sample $(\alpha=0.89)$. Higher scores on the GBMM scale denote greater mistrust in the medical community because of one's race/ethnicity.

\section{Medical Mistrust: Sexual Orientation}

Participants took a modified version of the GBMM scale (Thompson et al., 2004) to assess mistrust in the medical community based on one's sexual orientation. In line with previous research (Owens et al., 2007), the word "ethnic group" was reworded to "sexual orientation." The response categories were the same as the GBMM scale for race/ethnicity. An example item for the revised scale includes "People of my sexual orientation cannot trust doctors and health care workers." Cronbach's alpha for the scale among our study sample indicated high internal consistency $(\alpha=0.90)$. Higher scores on the revised GBMM scale indicated greater mistrust in the medical community based on one's sexual orientation.

\section{Analytic Strategy}

We first performed descriptive statistics on all study variables. In order to examine attitudes toward broad consent among each scenario and institution, we dichotomized the broad consent questions such that those who indicated they were "Not At All Willing" to provide broad consent were coded as 0 and those who were any other response category were coded as 1 ("Willing"). We then examined frequencies among each scenario and institution to assess general attitudes toward broad consent among YSMM.

Next, we conducted linear regression models to examine whether GBMM based on race/ethnicity or sexual orientation was associated with willingness to provide broad consent for each type of institution. We then averaged the willingness to provide broad consent among each scenario to create an average broad consent score for each institution, with higher scores denoting greater willingness to provide broad consent for that institution. Next, we ran two linear regression models among each institution, one for the GBMM scale based on race/ethnicity and one for the GBMM scale based on sexual orientation for a total of 10 models. Further, to examine if the association between GBMM and willingness to provide broad consent was more pronounced among YSMM of color we ran an additional 10 models that included an interaction term between race/ethnicity and the GBMM scales. Age, sexual orientation, race, highest level of education attained, and sexual orientation were included as covariates in all models. All statistical analyses were conducted using Stata statistical software version 15 (StataCorp, 2015).

\section{Results}

\section{Attitudes Toward Broad Consent Among Scenarios and Institutions}

Among the scenarios within each institution, willingness versus unwillingness to provide broad consent remained relatively consistent (e.g., around $82-85 \%$ willing in each category among current researchers). However, as one moved further away from university researchers toward pharmaceutical companies and government organizations, the less likely the participants would be willing to provide broad consent for any research scenario (see Table 1 for average willingness to provide consent for each institution type and scenario type). Participants were least likely to endorse willingness to provide broad consent for secondary research to pharmaceutical companies. Figure 1 displays the average percentages of willingness versus unwillingness that YSMM had toward providing broad consent to each of the research institutions. On average, YSMM were most willing to provide broad consent to the researcher from the study they were enrolled 
in $(85.3 \%)$, other researchers within the same university (82.4\%), and researchers at other universities (74.5\%). In contrast, YSMM were least willing to provide broad consent to government organizations (64.4\%) and pharmaceutical companies (53.8\%).

\section{Medical Mistrust and Attitudes Toward Broad Consent}

The linear regression models indicated that medical mistrust based on one's racial/ethnic identity or sexual orientation was associated with attitudes toward the use of broad consent among current researchers, researchers at other universities, government organizations, and pharmaceutical companies. The models for other researchers within the same university did not reach significance. Moreover, the interaction terms between GBMM and medical mistrust did not approach significance in any of the models. Thus, we present the results of the main effects models. See Tables 2, 3, 4, and 5 for results of the models for current researchers, researchers at other universities, government organizations, and pharmaceutical companies, respectively.

Table 2 Linear regression models for current researchers $(N=237)$

\begin{tabular}{lll}
\hline & Model 1 & Model 2 \\
\hline Age & $b(\mathrm{SE})$ & $b(\mathrm{SE})$ \\
Race & $-.02(.07)$ & $.02(.07)$ \\
White, non-Hispanic & Ref & Ref \\
Non-White, non-Hispanic & $.09(.14)$ & $-.01(.14)$ \\
Hispanic & $.18(.16)$ & $.10(.51)$ \\
Education & & \\
High School Diploma/GED & Ref & Ref \\
Some College or More & $.01(.94)$ & $.04(.13)$ \\
Sexual orientation & & \\
Gay & Ref & Ref \\
Bisexual & $-.30(.18)$ & $-.25(.18)$ \\
Other & $-.47(.20)^{*}$ & $-.47(.20)^{*}$ \\
GBMM ${ }^{\text {a }-R a c e / E t h n i c i t y ~}$ & $-.30(.07)^{* * *}$ & - \\
GBMM ${ }^{\mathrm{a}}$-Sexual Orienta- & - & $-.24(.07)^{* *}$ \\
tion & & \\
Intercept & $4.50(1.76)^{*}$ & $4.38(1.78)^{*}$ \\
Model fit statistics & & \\
& $F(7,229)=4.06^{* * *}$ & $F(7,229)=3.28^{* *}$ \\
& $R^{2}=.11$ & $R^{2}=.09$ \\
\hline
\end{tabular}

${ }^{*} p<.05 ; * * p<.01 ; * * * p<.0001$

${ }^{\mathrm{a}}$ Group-based Medical Mistrust Scale
Table 3 Linear regression models for researchers at different universities $(N=237)$

\begin{tabular}{|c|c|c|}
\hline & Model 1 & Model 2 \\
\hline & $b(\mathrm{SE})$ & $b(\mathrm{SE})$ \\
\hline Age & $-.03(.08)$ & $-.03(.08)$ \\
\hline \multicolumn{3}{|l|}{ Race } \\
\hline White, non-Hispanic & $\operatorname{Ref}$ & Ref \\
\hline Non-White, non-Hispanic & $-.03(.17)$ & $-.10(.17)$ \\
\hline Hispanic & $-.02(.19)$ & $-.08(.19)$ \\
\hline \multicolumn{3}{|l|}{ Education } \\
\hline High School Diploma/GED & Ref & $\operatorname{Ref}$ \\
\hline Some College or More & $-.02(.15)$ & $.00(.15)$ \\
\hline \multicolumn{3}{|l|}{ Sexual orientation } \\
\hline Gay & $\operatorname{Ref}$ & Ref \\
\hline Bisexual & $-.12(.22)$ & $-.08(.21)$ \\
\hline Other & $-.45(.24)$ & $-.43(.24)$ \\
\hline $\mathrm{GBMM}^{\mathrm{a}}$ —Race/Ethnicity & $-.25(.09)^{* *}$ & - \\
\hline $\mathrm{GBMM}^{\mathrm{a}}$-Sexual Orientation & - & $-.27(.08)^{* *}$ \\
\hline Intercept & $4.50(2.12)^{*}$ & $4.68(2.11)^{*}$ \\
\hline \multicolumn{3}{|l|}{ Model fit statistics } \\
\hline & $F(7,229)=2.09 *$ & $F(7,229)=2.55^{*}$ \\
\hline & $R^{2}=.06$ & $R^{2}=.07$ \\
\hline
\end{tabular}

* $p<.05 ; * * p<.01$

${ }^{\mathrm{a}}$ Group-based Medical Mistrust Scale

Table 4 Linear regression models for government organizations $(N=237)$

\begin{tabular}{|c|c|c|}
\hline & Model 1 & Model 2 \\
\hline & $b(\mathrm{SE})$ & $b(\mathrm{SE})$ \\
\hline Age & $.02(.09)$ & $.02(.09)$ \\
\hline \multicolumn{3}{|l|}{ Race } \\
\hline White, non-Hispanic & Ref & Ref \\
\hline Non-White, non-Hispanic & $-.08(.19)$ & $-.18(.18)$ \\
\hline Hispanic & $-.06(.21)$ & $-.15(.20)$ \\
\hline \multicolumn{3}{|l|}{ Education } \\
\hline $\begin{array}{l}\text { High School Diploma/ } \\
\text { GED }\end{array}$ & Ref & $\operatorname{Ref}$ \\
\hline Some College or more & $.06(.17)$ & $.08(.16)$ \\
\hline \multicolumn{3}{|l|}{ Sexual orientation } \\
\hline Gay & Ref & Ref \\
\hline Bisexual & $-.08(.24)$ & $-.04(.23)$ \\
\hline Other & $-.21(.26)$ & $-.115(.26)$ \\
\hline GBMM ${ }^{\mathrm{a}}$-Race/Ethnicity & $-.36(.10)^{* * *}$ & - \\
\hline $\begin{array}{l}\text { GBMM }^{\mathrm{a}}-\text { Sexual Orienta- } \\
\text { tion }\end{array}$ & - & $-.47(.09)^{* * *}$ \\
\hline Intercept & $2.91(2.33)$ & $3.46(2.27)$ \\
\hline \multicolumn{3}{|l|}{ Model fit statistics } \\
\hline & $F(7,229)=2.81 * *$ & $F(7,229)=4.90 * * *$ \\
\hline & $R^{2}=.08$ & $R^{2}=.13$ \\
\hline
\end{tabular}

${ }^{*} p<.05 ; * * p<.01 ; * * * p<.0001$

${ }^{\mathrm{a}}$ Group-based Medical Mistrust Scale 
Table 5 Linear regression models for pharmaceutical companies $(N=237)$

\begin{tabular}{|c|c|c|}
\hline & Model 1 & Model 2 \\
\hline & $b(\mathrm{SE})$ & $b(\mathrm{SE})$ \\
\hline Age & $-.02(.09)$ & $-.02(.09)$ \\
\hline \multicolumn{3}{|l|}{ Race } \\
\hline White, non-Hispanic & $R e f$ & Ref \\
\hline Non-White, non-Hispanic & $.11(.19)$ & $-.02(.18)$ \\
\hline Hispanic & $-.01(.21)$ & $-.12(.20)$ \\
\hline \multicolumn{3}{|l|}{ Education } \\
\hline $\begin{array}{l}\text { High School Diploma/ } \\
\text { GED }\end{array}$ & Ref & Ref \\
\hline Some College or More & $-.32(.17)$ & $-.29(.17)$ \\
\hline \multicolumn{3}{|l|}{ Sexual orientation } \\
\hline Gay & $\operatorname{Ref}$ & $\operatorname{Ref}$ \\
\hline Bisexual & $-.20(.24)$ & $-.15(.23)$ \\
\hline Other & $-.35(.26)$ & $-.32(.26)$ \\
\hline GBMM ${ }^{\mathrm{a}}$-Race/Ethnicity & $-.42(.10)^{* * *}$ & - \\
\hline $\begin{array}{l}\text { GBMM }^{\mathrm{a}}-\text { Sexual Orienta- } \\
\text { tion }\end{array}$ & - & $-.47(.09)^{* * *}$ \\
\hline Intercept & $4.32(2.34)$ & $4.66(2.30)^{*}$ \\
\hline \multicolumn{3}{|l|}{ Model fit statistics } \\
\hline & $F(7,229)=3.52 * *$ & $F(7,229)=4.74 * * *$ \\
\hline & $R^{2}=.10$ & $R^{2}=.13$ \\
\hline
\end{tabular}

${ }^{*} p<.05 ; * * p<.01 ; * * * p<.0001$

${ }^{\mathrm{a}}$ Group-based Medical Mistrust Scale

\section{Current Researchers}

Results indicated that both medical mistrust based on race/ ethnicity (Table 2, Model 1) and sexual orientation (Table 2,
Model 2) were associated with willingness to provide broad consent for secondary research among current researchers. Controlling for age, race, education, and sexual orientation, those with more medical mistrust based on race/ethnicity were less willing to provide broad consent to their current researchers $(b=-0.30, p<0.0001$; Table 2, Model $1)$. Similar results were found for those with more medical mistrust based on one's sexual orientation $(b=-0.24$, $p<0.01$; Table 2, Model 2). In addition, we found evidence to suggest that, as compared to young men who identify as gay, those who identify as a sexual orientation other than gay or bisexual were less willing to provide broad consent to their current researchers in models controlling for medical mistrust based on race/ethnicity $(b=-0.47, p<0.05$; Table 2 , Model 1) and medical mistrust based on sexual orientation $(b=-0.47, p<0.05$; Table 2, Model 2).

\section{Researchers at Different Universities}

Linear regression results indicated that greater medical mistrust based on one's race/ethnicity (Table 3, Model 1) and sexual orientation (Table 3, Model 2) were associated with willingness to provide broad consent to researchers at different universities. Controlling for age, race, education, and sexual orientation, those with more medical mistrust based on race/ethnicity were less willing to provide broad consent to their current researchers $(b=-0.25, p<0.01$; Table 3, Model $1)$. Similar results were found for those with more medical mistrust based on one's sexual orientation $(b=-0.28$, $p<0.01$; Table 3, Model 2).
Fig. 1 Average Percentages for Broad Consent by Institution

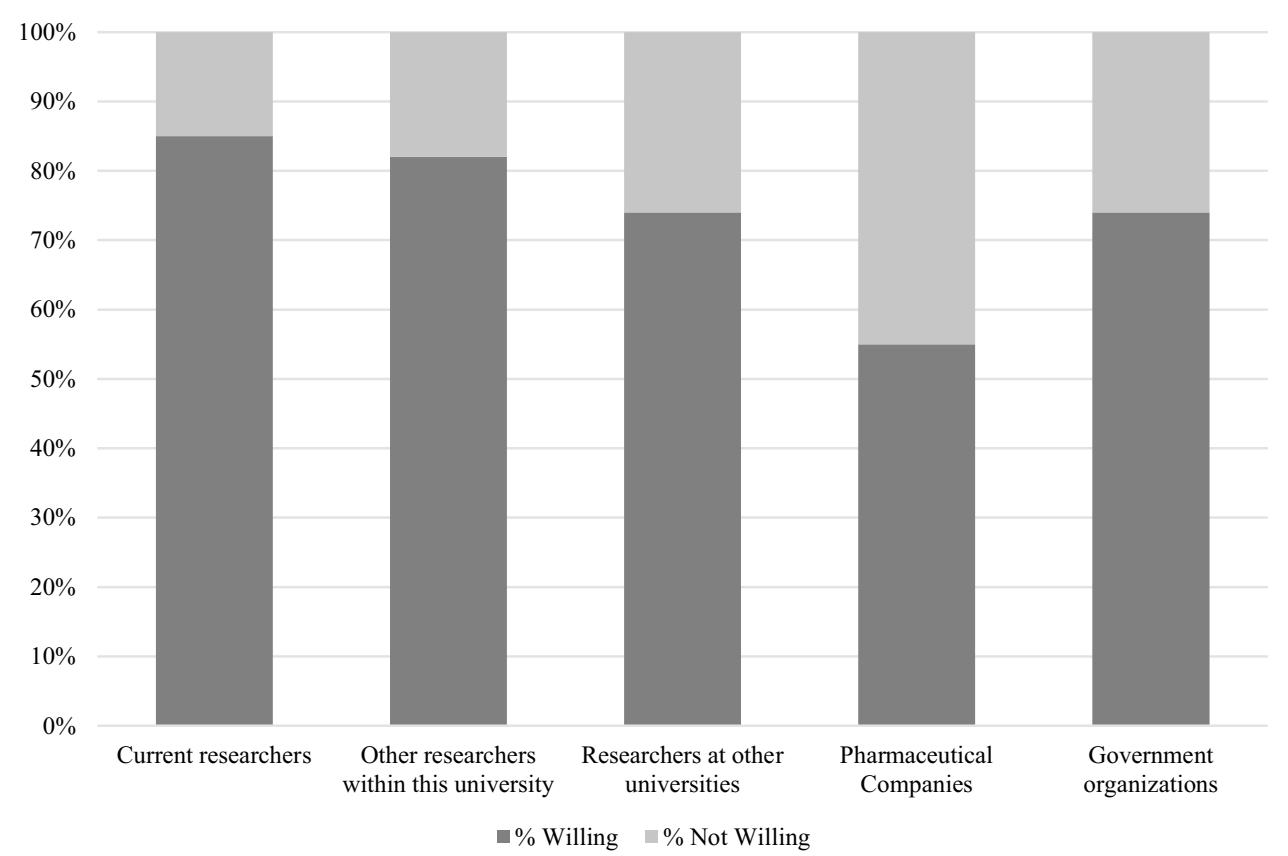




\section{Government Organizations}

For government organizations, results indicated that those who indicated more medical mistrust based on one's race/ethnicity were less willing to provide broad consent to government organizations $(b=-0.36, p<0.0001$; Table 4, Model 1$)$ controlling for age, race, education, and sexual orientation. The same trend was observed for medical mistrust based on sexual orientation ( $b=-0.47, p<0.0001$; Table 4, Model 2).

\section{Pharmaceutical Companies}

Results indicated that greater medical mistrust based on one's race/ethnicity $(b=-0.42, p<0.0001$; Table 5 , Model 1$)$ and sexual orientation $(b=-0.47, p<0.0001$; Table 5 , Model 2 ) was associated with a greater unwillingness to provide broad consent for secondary research with biospecimens to pharmaceutical companies.

\section{Discussion}

In this study, we sought to examine attitudes that YSMM hold toward the use of broad consent across a variety of secondary research scenarios types and institutions. Broadly speaking, we found that YSMM were generally willing to provide broad consent across a variety of secondary research topics. The majority of YSMM were likely to endorse providing broad consent across a variety of research scenarios (e.g., vaccine development, HIV-related research) if it was given to researchers that they trusted (i.e., the researchers of the study they were currently enrolled in) or other university-associated researchers. On the other hand, we found that YSMM were split, on average, in their willingness to provide broad consent to pharmaceutical companies and government organizations. Across the different research scenarios, the largest percentages of participants unwilling to provide broad consent were in the "understanding genetic differences between people" and "for finding a vaccination against common diseases" categories. These findings are in line with previous research on perspectives of broad consent in that the majority of the individuals are willing to provide broad consent for the use of their biospecimens in secondary research (Michie et al., 2011; Richter et al., 2018). One finding that was in contradiction to other research (Eaton et al., 2015) was that YSMM were generally likely to endorse providing consent for the use of their stored biospecimens in research related to HIV, but this willingness decreased as one moved to institutions farther away from their current researcher. These findings suggest that further consideration should be given to setting boundaries within the provision of broad consent to who can use stored biospecimens from YSMM in secondary research.
We also sought to explore the role that medical mistrust plays in the willingness to provide broad consent to each institution type. We found that medical mistrust played a role in willingness to give broad consent to current researchers, researchers at different universities, pharmaceutical companies, and government organizations, but not for other researchers within the same university. Our findings suggest that those with more medical mistrust based on one's racial/ethnic identity or sexual orientation are less willing to provide broad consent, on average, to current researchers, researchers at different organizations, pharmaceutical companies, or government organizations. These findings align with previous research that suggests that medical mistrust influences how racial/ethnic and/or sexual minority individuals engage and utilize health care (Eaton et al., 2015; Hoyt et al., 2012). It is important to note that the association between medical mistrust and willingness to give broad consent was stronger among pharmaceutical companies and government organizations. This finding suggests that YSMM who hold more medical mistrust based on their race/ethnicity or sexual orientation may be more cynical about pharmaceutical company and government research with their stored biospecimens. This finding has been prevalent among studies with ethnic/racial minorities (Garrison et al., 2016; Hiratsuka et al., 2012). For instance, a qualitative study with Alaska Native participants found that history and past experiences with the government and medical community led them to be more critical of participating in research that includes the storage of biospecimens (Hiratsuka et al., 2012). Further, in a large experimental survey examining patient attitudes toward participation in research involving stored human biospecimens Sanderson et al. (2017) found ethnic/racial minorities to be less likely to provide broad consent for the use of stored biospecimens as compared to their white counterparts. However, we did not find evidence to suggest that race/ethnicity moderated the association between medical mistrust and concerns pertaining to broad consent. This indicates that, at least in these data, overall medical mistrust may be salient for YSMM independent of race. Some research exists in support of this finding. For instance, in qualitative interviews with 56 SMM, Cahill et al. (2017) found that medical mistrust was a strong factor in willingness to uptake PrEP among both white YSMM and YSMM of color. Taken together, future research should utilize qualitative methods in order to further unpack and understand the attitudes that diverse samples of YSMM hold toward the use of broad consent, especially among specific entities that they may view as problematic (e.g., pharmaceutical companies).

There were important study limitations to consider. First, we recruited our participants from an ongoing cohort study based out of New York City, in which the participants have established a good relationship with the research team. Thus, our study sample may be more willing to participate 
in research than the more general population of YSMM. In addition, because participants were recruited from a wider cohort study, we were unable to ascertain if differences exist in willingness to provide broad consent across different racial/ethnic groups (e.g., Black Hispanic vs. Black non-Hispanic). In addition, the sample size within each racial/ethnic category was small and thus this study should be replicated with a larger, more diverse sample size to examine potential racial differences in the association between medical mistrust and broad consent that we may not have been able to detect. Moreover, our study was also limited in that we only assessed willingness to provide broad consent among a young adult sample. However, research suggests that generational differences may exist with respect medical mistrust such that older populations may harbor more mistrust than younger populations (Ford et al., 2013). In spite of these limitations, we are among the first to examine the ethical implications of the use of broad consent with stored biospecimens among YSMM and YSMM of color in particular. Future research should explore attitudes toward broad consent using a more diverse, research naïve sample of YSMM.

\section{Public Health Implications}

Medical mistrust continues to be a pressing issue in public health and medicine. Indeed, the legacy of mistrust, stemming from historical abuses both within and beyond the medical system, is reinforced and perpetuated in the form of ongoing mistreatment, stigma, and discrimination. As advances in biomedicine create opportunities to effect positive change, they also provide opportunities for researchers and IRBs to critically reflect on how new policies and guidelines are perceived, and how they ultimately affect, the populations that provide research data.

This study contributes to an increased awareness surrounding concerns that YSMM have over the use of broad consent for secondary research with their stored biological data. These implications are particularly salient for research conducted among populations that have experienced historical and ongoing social and economic marginalization, such as people of color and sexual minority people. This study suggests that researchers and IRB members should consider incorporating concerns of YSMM into study protocol surrounding the use of stored biospecimens in secondary research. Particularly, researchers may want to consider adopting measures that increase participants' comfort in utilizing stored biospecimens for secondary research purposes. For example, making specific efforts to ensure that participants understand the nature of their contribution and potential future contributions to science is one important way that researchers can work to engender trust and enhance participants' ability to exercise agency and feel confident in their decision to participate in research. Indeed, this may be especially critical for participants in ongoing longitudinal studies, for whom new applications or technologies may emerge during the course of their participation. Such measures can increase recruitment and retention efforts for critically important research, such as HIV cure research studies.

In a rapidly changing technological environment, it may be impossible to conceive of all the different ways data could be used in the future; however, being cognizant of these concerns and possible ramifications can help researchers to reduce mistrust among communities that have experienced stigma, discrimination and/or abuse in the medical establishment. In this sense, we are able to uphold our responsibility to protect participants while maximizing the ethical and innovative use of collected data to advance science.

Acknowledgements We would like to acknowledge the funding sources which was a pilot grant sponsored by the National Institute on Drug Abuse (\#1R25DA031608, Principal Investigator, Celia B. Fisher)

\section{Declarations}

Conflict of interest The authors declare that they have no conflict of interest.

Ethical Approval The study was approved by the New York University Institutional Review Board.

\section{References}

A Notice of Proposed Rule Making [NPRM] 2015 Summary. (2016, March). https://www.hhs.gov/ohrp/regulations-and-policy/regul ations/nprm-2015-summary/index.html.

Cahill, S., Taylor, S. W., Elsesser, S. A., Mena, L., Hickson, D., \& Mayer, K. H. (2017). Stigma, medical mistrust, and perceived racism may affect PrEP awareness and uptake in black compared to white gay and bisexual men in Jackson, Mississippi and Boston, Massachusetts. AIDS Care, 29(11), 1351-1358. https://doi.org/10. 1080/09540121.2017.1300633

Department of Homeland Security (DHS). (2017). Federal register federal policy for the protection of human subjects. Retrieved from https://www.gpo.gov/fdsys/pkg/FR-2017-01-19/pdf/2017-01058. pdf

De Souza, Y. G., \& Greenspan, J. S. (2013). Biobanking past, present and future: Responsibilities and benefits. AIDS, 27(3), 303-312. https://doi.org/10.1097/QAD.0b013e32835c1244

De Vries, R. G., Tomlinson, T., Kim, H. M., Krenz, C., Haggerty, D., Ryan, K. A., \& Kim, S. Y. H. (2016). Understanding the public's reservations about broad consent and study-by-study consent for donations to a biobank: Results of a national survey. PLOS ONE, 11. https://doi.org/10.1371/journal.pone.0159113

Eaton, L. A., Driffin, D. D., Kegler, C., Smith, H., Conway-Washington, C., White, D., \& Chauncey, C. (2015). The role of stigma and medical mistrust in the routine health care engagement of black men who have sex with men. American Journal of Public Health, 105(2), e75-e82. https://doi.org/10.2105/AJPH.2014.302322

Fisher, C. B., \& Layman, D. (2018). Genomics, big data, and broad consent: A new ethics frontier for prevention science. Prevention Science, 19(7), 871-879. https://doi.org/10.1007/s11121-018-0944-z 
Ford, C. L., Wallace, S. P., Newman, P. A., Lee, S.-J., \& Cunningham, W. E. (2013). Belief in AIDS-related conspiracy theories and mistrust in the government: Relationship with HIV testing among at-risk older adults. The Gerontologist, 53(6), 973-984. https:// doi.org/10.1093/geront/gns 192

Garrison, N. A., Sathe, N. A., Antommaria, A. H. M., Holm, I. A., Sanderson, S. C., Smith, M. E., McPheeters, M. L., \& Clayton, E. W. (2016). A systematic literature review of individuals' perspectives on broad consent and data sharing in the United States. Genetics in Medicine, 18(7), 663-671. https://doi.org/10.1038/ $\operatorname{gim} .2015 .138$

Grady, C., Eckstein, L., Berkman, B., Brock, D., Cook-Deegan, R., Fullerton, S. M., \& Wendler, D. (2015). Broad consent for research with biological samples: Workshop conclusions. American Journal of Bioethics, 15(9), 34-42.

Halkitis, P. N., Moeller, R. W., Siconolfi, D. E., Storholm, E. D., Solomon, T. M., \& Bub, K. L. (2013). Measurement model exploring a syndemic in emerging adult gay and bisexual men. AIDS and Behavior, 17, 662-673. https://doi.org/10.1080/15265161.2015. 1062162

Hiratsuka, V., Brown, J., \& Dillard, D. (2012). Views of biobanking among Alaska Native people: The role of community context. Progress in Community Health Partnerships: Research, Education, and Action, 6(2), 131-139. https://doi.org/10.1353/cpr.2012.0025

Hoyt, M. A., Nemeroff, C. J., Huebner, D. M., Rubin, L. R., Lee, J., \& Proeschold-Bell, R. J. (2012). HIV/AIDS-related institutional mistrust among multiethnic men who have sex with men: Effects on HIV testing and risk behaviors. Health Psychology, 31(3), 269-277. https://doi.org/10.1037/a0025953

Katz, R. V., Russell, S. L., Kressin, N. R., Green, B. L., Wang, M. Q., James, S. A., \& Claudio, C. (2006). The Tuskegee Legacy Project: Willingness of minorities to participate in biomedical research. Journal of Health Care for the Poor and Underserved, 17(4), 698-715. https://doi.org/10.1353/hpu.2006.0126

Kerath, S. M., Klein, G., Kern, M., Shapira, I., Witthuhn, J., Norohna, N., \& Taioli, E. (2013). Beliefs and attitudes towards participating in genetic research: A population-based cross-sectional study. BMC Public Health, 13. https://doi.org/10.1186/1471-2458-13-114

Menikoff, J., Kaneshiro, J., \& Pritchard, I. (2017). The common rule, updated. New England Journal of Medicine, 376(7), 613-615. https://doi.org/10.1056/NEJMp1700736
Michie, M., Henderson, G., Garrett, J., \& Corbie-Smith, G. (2011). "If I could in any small way help": motivations for and beliefs about sample donation for genetic research. Journal of Empirical Research on Human Research Ethics, 6(2), 57-70. https://doi.org/ 10.1525/jer.2011.6.2.57

Owens, G. P., Riggle, E. D., \& Rostosky, S. S. (2007). Mental health services access for sexual minority individuals. Sexuality Research and Social Policy, 4(3), 92-99. https://doi.org/10.1525/srsp. 2007.4.3.92

Richter, G., Krawczack, M., Lieb, W., Wolff, L., Schrieber, S., \& Buyx, A. (2018). Broad consent for health care-embedded biobanking: understanding and reasons to donate in a large patient sample. Genetics in Medicine, 20(1), 76-82. https://doi.org/10.1038/gim. 2017.82

Rothstein, M. A., Knoppers, B. M., \& Harrell, H. L. (2016). Comparative approaches to biobanks and privacy. Journal of Law, Medicine and Ethics, 44, 161-172. https://doi.org/10.1177/1073110516 644207

Sanderson, S. C., Brothers, K. B., Mercaldo, N. D., Clayton, E. W., Antommaria, A. H., \& Aufox, S. A. (2017). Public attitudes toward consent and data sharing in biobank research: A large multi-site experimental survey in the US. American Journal of Human Genetics, 100, 414-427. https://doi.org/10.1016/j.ajhg.2017.01. 021

StataCorp. (2015). Stata statistical software: Release 14. StatCorp LLC. Thompson, H. S., Valdimarsdottir, H. B., Winkel, G., Jandorf, L., \& Redd, W. (2004). The group-based medical mistrust scale: Psychometric properties and association with breast cancer screening. Prevention Medicine, 38(2), 209-281. https://doi.org/10.1016/j. ypmed.2003.09.041

Publisher's Note Springer Nature remains neutral with regard to jurisdictional claims in published maps and institutional affiliations. 